\title{
Solar Cell Surface Defects Detection based on Computer Vision
}

\author{
Xiaoliang Qian, Heqing Zhang, Huanlong Zhang, Yuanyuan Wu, Zhihua Diao, \\ Qing-E Wu, Cunxiang Yang*
}

School of Electrical and Information Engineering, Zhengzhou University of Light Industry, Zhengzhou, 450002, China

\begin{abstract}
Various types of defects exist in the solar cell surface because of some uncontrollable factors during the process of production. The solar cell surface defects detection is indispensable for the production of solar cell. The automatic defects detection methods based on computer vision have been widely used because of its convenience, real time and low cost. The state-of-the-art methods of solar cell surface defects detection based on computer vision are reviewed in this paper. Firstly, the typical defects of solar cell surface are summarized. Secondly, the state-of-the-art methods are classified into three categories: local scheme, global scheme and local-global scheme based methods, and separately introduced. Thirdly, the qualitative and exact evaluations of state-of-the-art methods are presented. The main contents of this paper and future development trends are summarized in the end.
\end{abstract}

Keywords: solar cell; surface defects detection; computer vision; feature extraction

(Submitted on July 25, 2017; Revised on August 30, 2017; Accepted on September 15, 2017)

(This paper was presented at the Third International Symposium on System and Software Reliability.)

(C) 2017 Totem Publisher, Inc. All rights reserved.

\section{Introduction}

Along with the worsening of environment and the decreasing of energy, the solar photovoltaic power generation has become one of the most attractive solutions [13]. Solar power generation has become more and more attractive for many countries because of its advantages of pollution-free, large reserves and convenient exploitation. Installed capacity of global solar will reach $74 \mathrm{GW}$ by 2019. The development trend of the installed capacity of global solar [23] in recent years is shown in Figure 1. However, various types of defects exist in the surface of solar cells because of some uncontrollable factors during the production process [1]. The service life and power generation efficiency of the solar cell is affected significantly due to the defects of solar cell surface $[12,27]$. Thus the solar cell surface defects detection is very important for the production of solar cells.

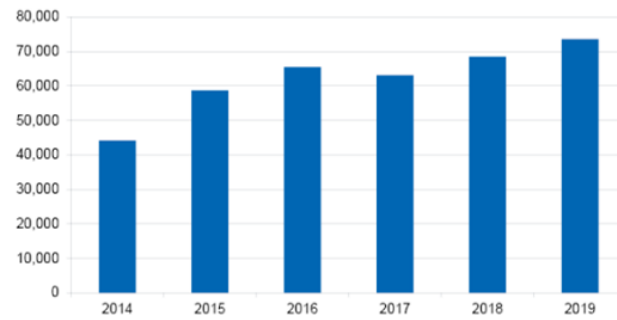

Figure 1. The development trend of global photovoltaic power capacity in recent years

With the development of computer vision, the defects detection technology of the solar cells surface is gradually transforming from artificial detection to automatic detection. Artificial detection includes human visual methods $[18,26]$ and

* Corresponding author.

E-mail address: qxl_sunshine@qq.com. 
artificial physical methods. The human visual detection is easily affected by individual factors and environmental factors. The existing artificial physical methods can be summarized as follows. Chen et al. [7] presented a detection method based on a noise solution. Sawyer et al. [25] introduced an idea that used laser scanning technology to detect defects. Dallas et al. [9] proposed a resonance ultrasonic vibrations technique for defects inspection. Zhang et al. [41] introduced a method based on optical deep-level transient spectroscopy. Wen et al. [37] detected surface defects through interferometric analysis of electronic speckle patterns.

Compared with the above artificial methods, the automatic detection methods based on computer vision are fast, economical, intelligent and easier for on-line detection [3,4]. Therefore, these methods have become one of the main developing directions of the solar cell surface defects detection and are widely used in the production of solar cells. The basic flow of solar cell surface defects detection based on computer vision is shown in Figure 2. Firstly, the solar cells are illuminated uniformly by the light source to capture high quality images through camera. Secondly, the captured images are imported into the image processing module for defects detection. Finally, the defects detection results are usually presented in the form of binary image.

The rest of the paper is organized as follows. The typical defects of solar cells surface are summarized in section 2. In section 3, the existing defects detection methods based on computer vision are classified into three categories, and respectively introduced. The qualitative and exact evaluations of state-of-the-art methods are presented in section 4 . The main contents of this paper and future development trends are given in section 5 .

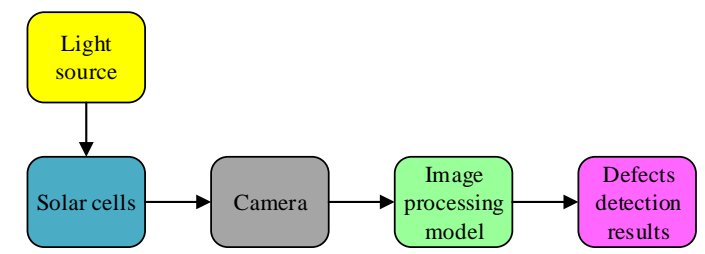

Figure 2. The basic flow of solar cell surface defects detection based on computer vision

\section{Typical defects of solar cells surface}

Various defects of solar cell surface may appear during the production of solar cells due to some uncontrollable factors, such as: manual errors, mechanical pressure caused by the machine breakdown, etc. [29,37]. The typical solar cell surface defects and their causes are shown in Table 1. Four kinds of typical defects in Table 1 are shown in Figure 3.

Table 1. The typical defects of solar cell surface
\begin{tabular}{|c|c|}
\hline Defects & Causes \\
\hline $\begin{array}{c}\text { Scratch } \\
\text { Broken gate }\end{array}$ & $\begin{array}{c}\text { Mechanical pressure, } \\
\text { Improper manipulation }\end{array}$ \\
\hline $\begin{array}{c}\text { Missing angle } \\
\text { Breakage }\end{array}$ & $\begin{array}{c}\text { Miscut, } \\
\text { Collision }\end{array}$ \\
\hline $\begin{array}{c}\text { Common spot } \\
\text { Fingerprint }\end{array}$ & $\begin{array}{c}\text { Hygiene problem, } \\
\text { Manual errors }\end{array}$ \\
\hline Blue spot & $\begin{array}{c}\text { Chemical reaction is not } \\
\text { uniform in the coating }\end{array}$ \\
\hline
\end{tabular}

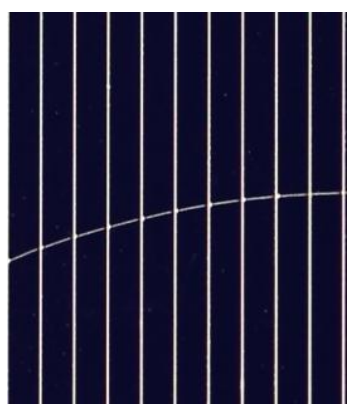

(a) Scratch

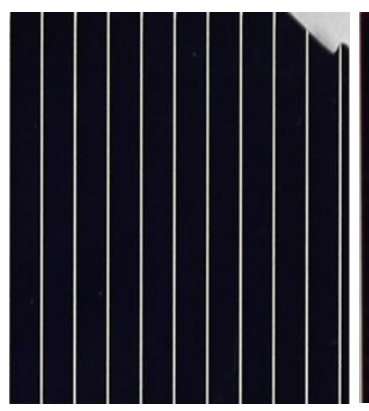

(b) Missing angle

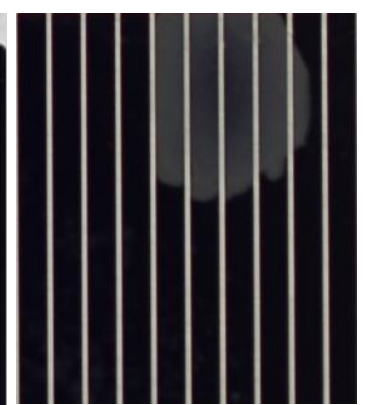

(c) Spot

Figure 3. Four kinds of typical defects

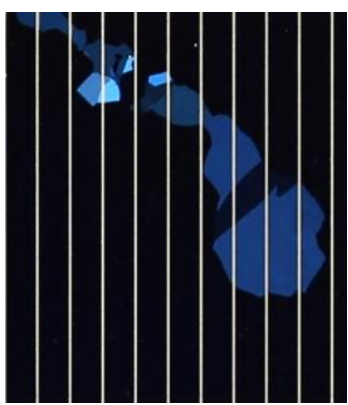

(d) Blue spots 


\section{State-of-the-art Methods}

The solar cell surface defects detection methods based on computer vision can be roughly divided into three categories: 1) local scheme based methods, 2) global scheme based methods, 3) local-global scheme based methods in which the local scheme and global scheme are jointly used for defects detection. The three kinds of methods and comparison of their characteristics will be introduced as follows.

\subsection{Defects detection based on local scheme}

A majority of existing methods can be classified into the first category, which mainly consists of two steps. Firstly, the local image features are extracted, such as: gradient, texture, local intensity contrast, etc. Secondly, the defective regions are segmented by threshold, clustering, etc. according to the local features. A brief introduction of some representative works of this kind of methods will be given as follows.

A local gradient features based method was proposed by Anwar et al. [5] for surface defects detection. Each region will be sharpened or smoothed by anisotropic diffusion according to its gradient value. Therefore, the defective regions are sharpened because their gradient value is obviously greater than the non-defective regions. In contrast, the non-defective regions are smoothed. The defective regions are thus highlighted in the new image obtained by aforementioned sharpening and smoothing operation. A difference image in which most of the non-defective regions are eliminated can be obtained by subtracting the new image from original image. Finally, simple threshold segmentation and morphological operations are imposed on the difference image to get the result image.

Tsai et al. [31] proposed a detection method which used the entropy of local gradient direction for defects detection. Firstly, the edge image is obtained through the edge detection of input image. Secondly, the entropy of gradient direction is calculated within each neighborhood of edge image for obtaining the entropy image due to the motivation that the gradient direction of edge within non-defective regions is more consistent than those within defective regions. Thirdly, the entropy image is smoothed by using the mean-shift algorithm, which can suppress the noises but reserve the edges. Finally, the smoothed entropy image is segmented by a simple threshold processing to get the binary image.

An effective method which combined the local gradient features with PSO (Particle Swarm Optimization) algorithm was proposed by Aghamohammadi et al. [2]. As shown in Figure 4, the PSO algorithm is used for edge detection to get the edge image. Secondly, the texture map is obtained by the texture feature extraction of the edge image. Thirdly, the crack feature extraction is carried out by the gradient computation of texture map. Finally, the cracks and grid lines are extracted from the input image according to the crack feature map, and sent to the fuzzy logic system for the identification of cracks.

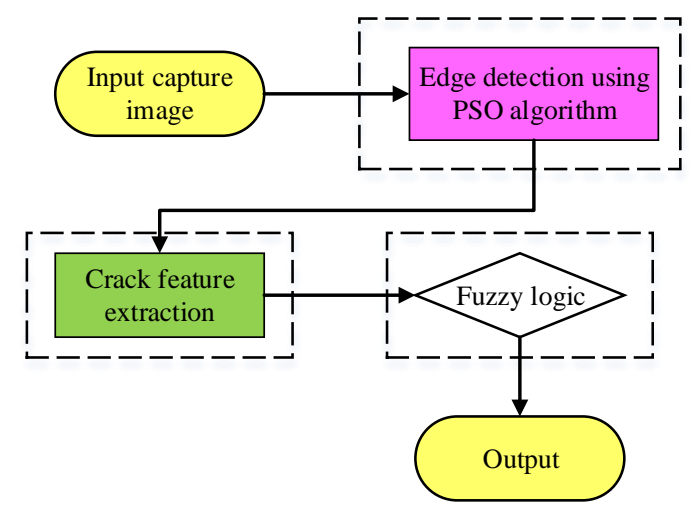

Figure 4. Flowchart of Aghamohammadi et al. [2]

Xu et al. [38] introduced an Otsu segmentation based approach for defects inspection. First of all, the surface image of solar cell array is smoothed by a Gaussian filter. Then, the edge detection and image segmentation technique is imposed on the smoothed image for the segmentation of single solar cell. Thirdly, the single solar cell surface image is divided into background and foreground by Otsu segmentation. Fourthly, the lines detection of segmented image is implemented through Hough transform. Finally, the detection result is obtained by subtracting the lines detection result from smoothed image. The detection procedure is shown in Figure 5. 
A defects detection method which combined the local descriptors with SVM (support vector machine) was proposed by Demant et al. [11]. First of all, a set of labeled samples generated by artificial insertion of cracks is required for training SVM. The rich diversity of crack samples is necessary for the representativeness of sample set. Secondly, a set of local descriptors is used to extract the features of training samples and captured images. The feature vectors of training samples are used to train the SVM in training mode. The surface cracks are recognized by importing the feature vectors of captured image into the SVM in testing mode. Other excellent works based on local scheme include Tsai et al. [34], Fu et al. [13], Takahashi et al [28], Fuyuki et al [14], Demant et al [10] etc.

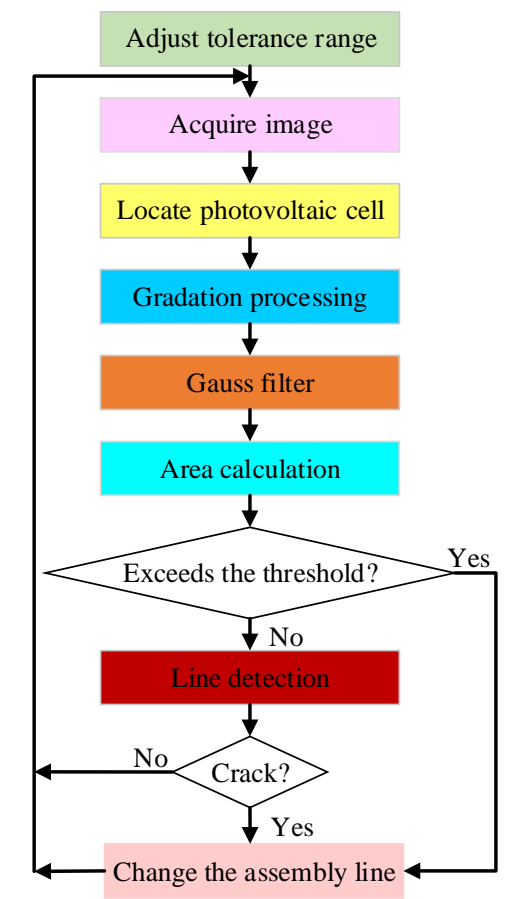

Figure 5. Detection procedure of $\mathrm{Xu}$ et al. [38]

\subsection{Defects detection based on global scheme}

The implementation of this kind of methods mainly consisted of two steps. First of all, the input image is transformed integrally by Fourier transform, Wavelet transform, and matrix factorization etc. Then, the detecting result can be obtained by inverse transformation of intermediate results, which are gotten by the proper processing of transforming image. Some of the representative works of this kind of methods are briefly introduced in the following paragraphs.

A Fourier transform based method was presented by Tsai et al. [32]. First of all, the spectral image is obtained through the Fourier transform of input image. Secondly, a new spectral image is generated by setting the possible frequency components associated with defective regions to zero. Thirdly, the "defect-free" image is gotten by applying the inverse Fourier transform to the new spectral image. Finally, the defective regions are detected by subtracting the "defect-free" image from input image.

A similar method which adopts wavelet-based discriminant measure for defects inspection was proposed by Li et al. [20]. The wavelet multiresolution decomposition of input image is firstly implemented. The wavelet coefficients of each resolution are regarded as the features of input image. The difference between coefficients of two successive resolutions is used for weights of features. The defects are finally extracted from the background according to the weighted features.

Applying matrix decomposition for defects detection is another classical global based scheme. A SVD (Singular Value Decomposition) based method was proposed by Lu et al. [21]. Firstly, the SVD of original image matrix is implemented. Then, the singular values, which represent the background information, are eliminated from the diagonal matrix. The final detection result is reconstructed from the remainder singular values. 
A detection method [39] based on low rank matrix recovery is similar to [21]. The original image matrix is decomposed into the sum of a low rank matrix, which implies the homogeneous background texture, and a sparse matrix, which represents the irregular defects. The resultant image is finally derived from the sparse matrix.

Yen et al. [40] presented an efficient method based on auto-thresholding and projection approach for inspecting busbar and finger defects. As shown in Figure 6, the median filter is first used for suppressing the impulse noises. Secondly, a binary image is obtained by applying the auto-thresholding technique to the denoised image. Then, a projection method [19] is cited for identifying the busbars and fingers. The vertical scanning detection is implemented for busbars inspection after the localization of busbar regions. The scanning pixels of which gray-scale values are zero are marked green in the captured image for the visualization of busbar defects. The procedure of fingers inspection is similar to the busbars inspection. Other relevant works of using global scheme for surface defects detection include Brooks et al. [6], Mahdavipour et al. [22], Teo et al. [30], Wang [35] etc.

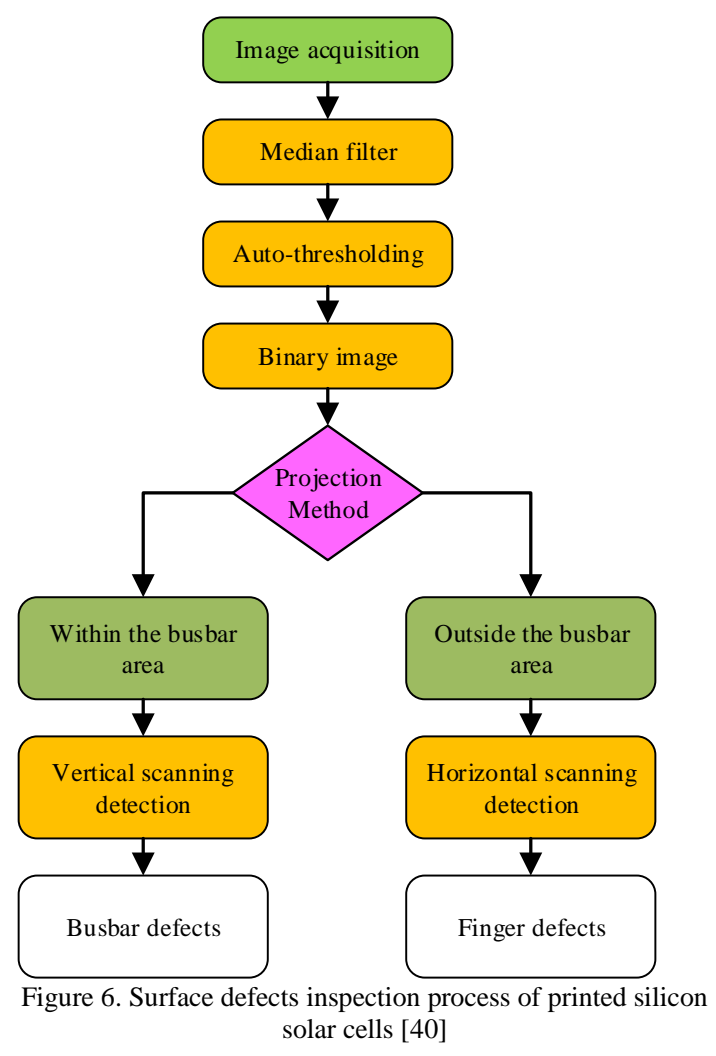

\subsection{Defects detection based on local-global scheme}

The local-global scheme based methods are presented in this section. The core idea of this kind of methods is combining local scheme with global scheme. For instance, the local scheme and global scheme are jointly used through machine learning, such as, ICA (Independent Component Analysis), deep learning, etc. Some of typical works are briefly introduced as follows.

Ko et al. [17] proposed a method in which the local difference and global morphological operations are combined for defects detection. As shown in Figure 7, the histogram equalization is firstly used for contrast enhancement of input image. Secondly, the gray scale difference between the defective regions and non-defective regions is enlarged by applying the anisotropic diffusion. The difference between the original image and smoothed image will highlight the defective regions. The initial result is obtained by using a simple binary thresholding. The morphological operations and particle filter which are separately used for filling the gaps and removing the isolate outliers are applied to the post-processing of initial result.

An ICA based method is proposed by Tsai et al. [33] for defects localization. In training stage, the defect-free images are divided into a set of sub-images, which is used for training the basis components through ICA algorithm. In the testing stage, the input image is projected onto the set of basis components for obtaining its feature matrix. The reconstruction 
image of input image is derived from the basis components and the feature matrix. The difference of the input image and reconstruction image is considered as the evaluation metric for defects detection.

Recently, Wang et al. [36] introduced a deep learning based method. In the training stage, first of all, an unsupervised layer-wise training method is used for pre-training the DBN (deep belief networks). Then, the weights of DBN are fine tuned by the defect-free samples. In the testing stage, the captured image is imported into the DBN to obtain the constructed image. The difference of the test image and the reconstructed image is used to determine whether or not the test image is a defective image. Other relevant works of using local-global scheme for surface defects detection include Gong et al. [15], Kim et al. [16], etc.

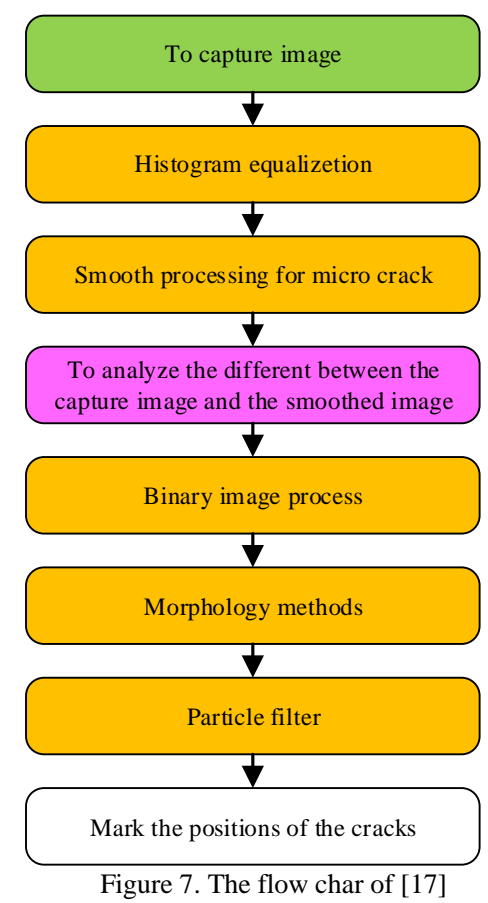

\subsection{Comparison of three kinds of methods}

The comparison of aforementioned three kinds of methods is shown in Table 2. The local scheme based methods are good at detection of cracks and broken bars. The global scheme based methods usually apply to multi-defects occasions, especially strip defects. The performance of local-global scheme based methods is more balanced for various defects.

Table 2. Comparison of three kinds of methods

\begin{tabular}{|c|c|c|c|}
\hline General description & $\begin{array}{l}\text { Local scheme } \\
\text { based methods }\end{array}$ & $\begin{array}{l}\text { Global scheme } \\
\text { based methods }\end{array}$ & $\begin{array}{l}\text { Local-global scheme } \\
\text { based methods }\end{array}$ \\
\hline Main idea & $\begin{array}{c}\text { Defective areas are } \\
\text { localized by segmentation } \\
\text { techniques according to } \\
\text { local features. }\end{array}$ & $\begin{array}{l}\text { The captured image is integrally transformed. The } \\
\text { transformed image is properly processed and } \\
\text { inversely transformed to obtain the detection results. }\end{array}$ & $\begin{array}{l}\text { The local and global } \\
\text { schemes are jointly used } \\
\text { for defects detection. }\end{array}$ \\
\hline Features & $\begin{array}{l}\text { Good at detection of } \\
\text { crack, broken bar. }\end{array}$ & $\begin{array}{l}\text { Apply to multi-defects occasions, especially for } \\
\text { strip defects. }\end{array}$ & $\begin{array}{l}\text { Balanced performance for } \\
\text { various defects. }\end{array}$ \\
\hline
\end{tabular}

\section{Evaluation}

\subsection{Datasets}

Up to now, all the published works use their own datasets for evaluation; however, all of the datasets are not publicly available. The existed datasets can be classified into two categories: 1) include multiple types of defects, 2) only microcracks included, and will be separately reviewed as follows. 
The first kind of datasets is used to evaluate the overall performance of detection methods. A dataset containing 323 test images was proposed by Tsai et al. [32], of which 308 test images were defect-free images and 15 test images were defective images containing micro-cracks, breaks, and finger-interruption. Another dataset containing 96 test images was proposed by $\mathrm{Li}$ et al. [20], of which 50 were defect-free images and 46 were defective images containing 13 contaminant images, 8 finger-interruption images and 25 saw-mark images. Qian et al. [24] presented a dataset which consists of 60 defect-free images and 40 defective images.

The second kind of datasets is specifically used for evaluating the capability of detecting micro-cracks since the microcracks detection is one of the most important tasks of solar cell surface defects detection. Anwar et al. [5] introduced a dataset which contains 313 defect-free images and 287 defective images. A dataset containing 2000 test images was proposed by Chiou et al. [8], of which 1967 test images were defect-free images and 33 test images were defective images. Another dataset proposed by Mahdavipour et al. [22] contained 100 test images, and all of images contained micro-cracks.

\subsection{Qualitative Evaluation}

A majority of published works $[8,20,24,32]$ evaluated their methods in this way. Qualitative evaluation only focuses on the capability that correctly decides whether or not the defects are existed. The detection accuracy is defined as:

$$
\text { Accuracy }=\frac{N_{T}}{N}
$$

where $N$ denotes the number of all the test images, $N_{\mathrm{T}}$ denotes the number of test images which are correctly identified. The qualitative detected results of $([8,20,24,32])$ are shown in Table 3. It's worth noting that the statistics of the listed 4 methods are cited from their papers because their source codes and datasets are not shared.

As shown in Table 3, the detection accuracy of the listed 4 methods reaches or closes to $100 \%$. As a matter of fact, the performance of state-of-the-art methods is comparable with each other in terms of qualitative evaluation if the difference of testing datasets is ignored.

Table 3. Comparison of qualitative detection results

\begin{tabular}{|l|c|c|c|c|}
\hline \multirow{2}{*}{ Sample types } & \multirow{2}{*}{$\begin{array}{c}\text { Number of } \\
\text { samples }\end{array}$} & \multicolumn{2}{|c|}{ Detection results } & \multirow{2}{*}{ Defective } \\
\cline { 3 - 4 } & $\begin{array}{c}\text { Defect- } \\
\text { free }\end{array}$ & \\
\hline Defect-free[20] & 50 & 0 & 50 & \multirow{2}{*}{$98.96 \%$} \\
\hline Defective[20] & 46 & 45 & 1 & \\
\hline Defect-free[24] & 60 & 1 & 59 & \multirow{2}{*}{$99.00 \%$} \\
\hline Defective[24] & 40 & 40 & 0 & \multirow{2}{*}{$99.85 \%$} \\
\hline Defect-free[8] & 1967 & 0 & 1967 & \multirow{2}{*}{$100 \%$} \\
\hline Defective[8] & 33 & 30 & 3 & \\
\hline Defect-free[32] & 308 & 0 & 308 & \\
\hline Defective[32] & 15 & 15 & 0 & \\
\hline
\end{tabular}

\subsection{Exact evaluation}

A few published works [5,24] adopted this evaluation mode. Unlike qualitative evaluation, the capability that precisely localize the defective regions is exactly quantified by calculating $\operatorname{Precission}(P), \operatorname{Recall}(R)$ and $F$-measure $(F)$ which can be formulated as:

$$
\begin{aligned}
& P=\frac{R_{d g}}{R_{d}} \\
& R=\frac{R_{d g}}{R_{g}} \\
& F=2 \frac{P \times R}{P+R}
\end{aligned}
$$

where $R_{d}$ denotes the detected defective regions, $R_{g}$ denotes the human-marked defective regions which are considered as ground truth of defects, $R_{d g}$ denotes the intersection of $R_{d}$ and $R_{g}$. The exact detected results of $([5,24])$ are shown in Table 4. The statistics of the listed 2 methods are also cited from their papers. 
As shown in Table 4, the overall performance of [24] is significantly superior than [5] if the difference of testing datasets is ignored.

Table 4. Comparison of Exact detection results

\begin{tabular}{|c|c|c|c|}
\hline Method & Precision & Recall & F-measure \\
\hline Anwar et al.[5] & 0.046 & 0.719 & 0.086 \\
\hline Qian et al.[24] & 0.867 & 0.705 & 0.778 \\
\hline
\end{tabular}

\section{Conclusions}

The state-of-the-art methods of solar cell surface defects detection based on computer vision are reviewed in this paper. The typical defects of solar cell surface are firstly introduced. Then, the state-of-the-art methods are classified into three categories and introduced separately. Qualitative and exact evaluations of several state-of-the-art works are presented in the end.

In our opinion, maybe the weakly-supervised deep learning is a promising research directions. The traditional deep neural networks have a strong ability for feature extraction; however, a lot of human annotated samples that always come with a heavy cost are required. The weakly-supervised deep learning which uses a large number of weak labels and a small amount of strong labels for pre-training and fine-tuning separately may be a good choice for effective defects detection. The weak labels only include the information that whether or not the defects are existed. The strong labels precisely mark the defective regions.

\section{Acknowledgements}

This work is supported by the National Science Foundation of China under Grants (No: 61501407, 61503173, 61603350), National 973 Program (No. 613237), Major Science and Technology Projects of Henan Province (No. 161100211600), Henan Province Outstanding Youth on Science and Technology Innovation (No. 164100510017), Key research project of Henan Province Universities (No: 15A413006), Key Science and Technology Program of Henan Province (No: 162102110118, 172102210062), Doctor fund project of Zhengzhou University of Light Industry (No: 2014BSJJ016, 2016BSJJ022), Youth Backbone Teacher Training Program of Henan Province Universities (2016GGJS-088), Science and technology innovation fund project of graduate student of Zhengzhou University of Light Industry(NO: 2016026).

\section{References}

1. M. Abbott, P. Cousins, F. Chen, and J. Cotter, "Laser-induced Defects in Crystalline Silicon Solar Cells," in Proceedings of the Photovoltaic Specialists Conference, pp. 1241-1244, Florida, USA, January 2005.

2. A. Aghamohammadi, A. Prabuwono, S. Sahran, and M. Mogharrebi, "Solar Cell Panel Crack Detection Using Particle Swarm Optimization Algorithm," in Proceedings of the International Conference on Pattern Analysis and Intelligent Robotics, pp. 160164, Putrajaya, Malaysia, June 2011.

3. K. Agroui, M. Pellegrino, and F. Giovanni, "Analysis Techniques for Photovoltaic Modules Based on Amorphous Solar Cells," Arabian Journal for Science and Engineering, vol. 42, no. 1, pp. 375-381, January 2017.

4. A. V. Andonova, G. Dobrikov, I. Zhivkov, and B. Bonev, "Degradation Detection as a Result of Ageing Organic Solar Cells by Thermography," in Proceedings of the Electronic System-Integration Technology Conference, pp. 1-4, Grenoble, France, September 2016.

5. S. A. Anwar and M. Z. Abdullah, "Micro-crack Detection of Multicrystalline Solar Cells Featuring an Improved Anisotropic Diffusion Filter and Image Segmentation Technique," Eurasip Journal on Image \& Video Processing, vol. 2014, no. 1, pp. 117, December 2014.

6. W. S. M. Brooks, D. A. Lamb, and S. J. C. Irvine, "IR Reflectance Imaging for Crystalline Si Solar Cell Crack Detection," IEEE Journal of Photovoltaics, vol. 5, no. 5, pp. 1271-1275, September 2015.

7. X. Y. Chen, A. Pedersen, O. G. Helles $\varnothing$, and A. D. V. Rheenen, "Electrical Noise of Laser Diodes Measured over a Wide Range of Bias Currents," Microelectronics Reliability, vol. 40, no. 11, pp. 1925-1928, November 2000.

8. Y. C. Chiou, J. Z. Liu, and Y. T. Liang, "Micro Crack Detection of Multi-crystalline Silicon Solar Wafer Using Machine Vision Techniques," Sensor Review, vol. 31, no. 2, pp. 154-165, Number 2011.

9. W. Dallas, O. Polupan, and S. Ostapenko, "Resonance Ultrasonic Vibrations for Crack Detection in Photovoltaic Silicon Wafers," Measurement Science \& Technology, vol. 18, no. 3, pp. 852-858, February 2007.

10. M. Demant, S. Rein, J. Krisch, S. Schoenfelder, and C. Fischer, "Detection and Analysis of Micro-cracks in Multi-crystalline Silicon Wafers during Solar Cell Production," in Proceedings of the Photovoltaic Specialists Conference (PVSC), pp. 001641001646, Santorini, USA, June 2011.

11. M. Demant, T. Welschehold, M. Oswald, S. Bartsch, T. Brox, S. Schoenfelder, et al., "Microcracks in Silicon Wafers I: Inline Detection and Implications of Crack Morphology on Wafer Strength," IEEE Journal of Photovoltaics, vol. 6, no. 1, pp. 1-10, 
January 2016.

12. S. Duenas, E. Perez, H. Castan, and H. Garcia, "The Role of Defects in Solar Cells: Control and Detection Defects in Solar Cells," in Proceedings of the Electron Devices (CDE), pp. 301-304, Valladolid, Spain, February, 2013.

13. Z. Fu, Y. Zhao, Y. Liu, and Q. Cao, "Solar Cell Crack Inspection by Image Processing," in Proceedings of the Business of Electronic Product Reliability and Liability, pp. 77-80, Shanghai, China, April 2004.

14. T. Fuyuki and A. Kitiyanan, "Photographic Diagnosis of Crystalline Silicon Solar Cells Utilizing Electroluminescence," Applied Physics A Materials Science \& Processing, vol. 96, no. 1, pp. 189-196, July 2009.

15. F. Gong, X. W. Zhang, and H. Sun, "Detection System for Solar Module Surface Defects Based on Constrained ICA Model and PSO Method," Acta Optica Sinica, vol. 32, no. 4, pp. 169-177, April 2012.

16. G. B. Kim, "Micro Defect Detection in Solar Cell Wafer Based on Hybrid Illumination and Near-infrared Optics," in Proceedings of the Control Conference (ASCC), pp. 1-5, Istanbul, Turkey, June 2013.

17. S. S. Ko, C. S. Liu, and Y. C. Lin, "Optical Inspection System with Tunable Exposure Unit for Micro-crack Detection in Solar Wafers," Optik - International Journal for Light and Electron Optics, vol. 124, no. 124, pp. 4030-4035, October 2013.

18. D. Lausch, T. Mehl, K. Petter, A. S. Flø, I. Burud, and E. Olsen, "Classification of Crystal Defects in Multicrystalline Silicon Solar Cells and Wafer Using Spectrally and Spatially Resolved Photoluminescence," Journal of Applied Physics, vol. 119, no. 5, pp. 223-136, February 2016.

19. S. W. Lee, D. J. Lee, and H. S. Park, "A New Methodology for Gray-scale Character Segmentation and Recognition," IEEE Transactions on Pattern Analysis and Machine Intelligence, vol. 18, no. 10, pp. 1045-1050, October 1996.

20. W. C. Li and D. M. Tsai, "Wavelet-based Defect Detection in Solar Wafer Images with Inhomogeneous Texture," Pattern Recognition, vol. 45, no. 2, pp. 742-756, February 2012.

21. C. J. Lu and D. M. Tsai, "Automatic Defect Inspection for LCDs Using Singular Value Decomposition," International Journal of Advanced Manufacturing Technology, vol. 25, no. 1-2, pp. 53-61, January 2005.

22. Z. Mahdavipour and M. Z. Abdullah, "Micro-crack Detection of Polycrystalline Silicon Solar Wafer," Iete Technical Review, vol. 32, no. 6, pp. 1-7, April 2015.

23. C. Network, "http://www.cableabc.com/," May 2016.

24. X. L. Qian, H. Q. Zhang, H. L. Zhang, Z. D. He, and C. X. Yang, "Solar Cells Surface Defect Detection Based on Visual Saliency," Chinese Journal of Scientific Instrument, vol. 38, no. 7, pp. 1570-1578, July 2017.

25. D. E. Sawyer and H. K. Kessler, "Laser Scanning of Solar Cells for the Display of Cell Operating Characteristics and Detection of Cell Defects," IEEE Transactions on Electron Devices, vol. 27, no. 4, pp. 864-872, April 1980.

26. S. Spataru, P. Hacke, and D. Sera, "Automatic Detection and Evaluation of Solar Cell Micro-cracks in Electroluminescence Images Using Matched Filters," in Proceedings of the IEEE Photovoltaic Specialists Conference, pp. 1602-1607, Portland, USA, June 2016.

27. R. Stephan, I. E. Reis, K. Wolfram, F. M. Aylin, S. Jonas, B. Herfried, et al., "Research on Efficiency Limiting Defects and Defect Engineering in Silicon Solar Cells - Results of the German Research Cluster SolarFocus," Physica Status Solidi, vol. 8, no. 3, pp. 733-738, March 2011.

28. Y. Takahashi, Y. Kaji, A. Ogane, and Y. Uraoka, "-"Luminoscopy"-Novel Tool for the Diagnosis of Crystalline Silicon Solar Cells and Modules Utilizing Electroluminescence," in Proceedings of the Photovoltaic Energy Conversion, pp. 924-927, Waikoloa, USA, May 2006.

29. T. W. Teo and M. Z. Abdullah, "In-line Photoluminescence Imaging of Crystalline Silicon Solar Cells for Micro-crack Detection," in Proceedings of the Imaging Systems and Techniques, pp. 66-70, Chania, Greece, October 2016.

30. T. W. Teo, Z. Mahdavipour, and M. Z. Abdullah, "High-speed Micro-crack Detection of Solar Wafers with Variable Thickness," in Proceedings of the Imaging Systems and Techniques (IST), pp. 237-241, Santorini, Greece, October 2014.

31. D. M. Tsai and J. Y. Luo, "Mean Shift-Based Defect Detection in Multicrystalline Solar Wafer Surfaces," IEEE Journals \& Magazines, vol. 7, no. 1, pp. 125-135, February 2011.

32. D. M. Tsai, S. C. Wu, and W. C. Li, "Defect Detection of Solar Cells in Electroluminescence Images Using Fourier Image Reconstruction," Solar Energy Materials \& Solar Cells, vol. 99, no. 99, pp. 250-262, April 2012.

33. D. M. Tsai, S. C. Wu, and W. Y. Chiu, "Defect Detection in Solar Modules Using ICA Basis Images," IEEE Transactions on Industrial Informatics, vol. 9, no. 1, pp. 122-131, February 2013.

34. D. M. Tsai, G. N. Li, W. C. Li, and W. Y. Chiu, "Defect Detection in Multi-crystal Solar Cells Using Clustering with Uniformity Measures, " Advanced Engineering Informatics, vol. 29, no. 3, pp. 419-430, August 2015.

35. N. Wang, "Silicon Solar Cell Based on Visual Detection Method," Baoding, Agricultural University of Hebei, May 2014.

36. X. B. Wang, J. Li, M. H. Yao, W. X. He, and Y. T. Qian, "Solar Cells Surface Defects Detection Based on Deep Learning," PR\&AI, vol. 27, no. 6, pp. 517-523, June 2014.

37. T. K. Wen and C. C. Yin, "Crack Detection in Photovoltaic Cells by Interferometric Analysis of Electronic Speckle Patterns," Solar Energy Materials \& Solar Cells, vol. 98, no. 5, pp. 216-223, January 2011.

38. P. Xu, W. J. Zhou, and M. R. Fei, "Detection Methods for Micro-cracked Defects of Photovoltaic Modules Based on Machine Vision," in Proceedings of the International Conference on Cloud Computing, pp. 609-613, Shenzhen, China, November 2014.

39. M. H. Yao, J. Li, and X. B. Wang, "Solar Cell Surface Defects Detection Using RPCA Method," Chinese Journal of Computers, vol. 36, no. 9, pp. 1943-1952, September 2013.

40. H. N. Yen and Y. J. Sie, "Machine Vision System for Surface Defect Inspection of Printed Silicon Solar Cells," in Proceedings of the Consumer Electronics, pp. 422-424, Tokyo, Japan, October 2012.

41. X. Zhang, J. Hu, Y. Wu, and F. Lu, "Direct Observation of Defects in Triple-junction Solar Cell by Optical Deep-level Transient Spectroscopy," Journal of Physics D Applied Physics, vol. 42, no. 14, pp. 145401-145405, July 2009. 